\title{
Ética na decisão terapêutica em condições de prematuridade extrema
}

Andrezza de Lemos Bezerra ${ }^{1}$, Giselle Machado Magalhães Moreno ${ }^{2}$, Adriana Carla Costa Ribeiro Clementino ${ }^{3}$, Anna Paula Chaves Chagas ${ }^{4}$, Isis Suruagy Moura ${ }^{5}$, Josimário Silva ${ }^{6}$

\section{Resumo}

Os limites de viabilidade na prematuridade e a sobrevivência com mínimo de sequela são temas atuais de grande importância ética. A prematuridade extrema pode resultar em inúmeras sequelas e danos crônicos que limitam a funcionalidade. Ao se optar pelo prolongamento da vida de recém-nascidos muito imaturos, há maior exposição do prematuro e da família a situações de dor, desconforto e estresse. A presente revisão trata dos aspectos éticos relacionados a essa tomada de decisão considerando o limite de viabilidade e as sequelas neuropsicomotoras decorrentes da prematuridade extrema. Atualmente, a questão gira em torno da importância da valorização não só da sobrevivência desses prematuros, mas também da manutenção de sua qualidade de vida, uma vez que são mais predispostos a sequelas em curto, médio e longo prazos.

Palavras-chave: Prematuro. Ética. Recém-nascido de peso extremamente baixo ao nascer. Desenvolvimento infantil.

\section{Resumen}

\section{Ética en las decisiones de tratamiento en condiciones de prematuridad extrema}

Los límites de la viabilidad de la prematuridad y la supervivencia con secuelas mínimas son las principales cuestiones éticas de actualidad. La prematuridad extrema puede dar lugar a numerosas secuelas y daños crónicos que limitan la funcionalidad. Cuando se opta por la prolongación de la vida de los recién nacidos muy inmaduros, aumenta la exposición del prematuro y de la familia a situaciones de dolor, malestar y estrés. Esta revisión discute los temas éticos relacionados con esta toma de decisión teniendo en cuenta el límite de la viabilidad y las secuelas neuropsicomotoras como consecuencia de la prematuridad extrema. En la actualidad, la cuestión gira en torno a la importancia de valorar no sólo la supervivencia de los bebés prematuros, sino también el mantenimiento de su calidad de vida, ya que son más propensos a las secuelas en el corto, mediano y largo plazo.

Palabras-clave: Prematuro. Ética. Recién nacido con peso al nacer extremadamente bajo. Desarollo infantil

\section{Abstract \\ Ethics related to therapeutic decision in cases of extreme prematurity}

Limits of viability for premature infants and survival with minimal consequences are current themes of great importance for ethics and bioethics. Extreme prematurity may result in many consequences and chronic injuries that limit the infant functionality. When prolongation of a very immature newborn's life is opted, there is a greater exposure of premature infants and their family to pain, distress and physiological stress situations. The present review approaches ethical aspects related to this decision-making, considering the limit of viability and neuromotor sequelae derived from extreme prematurity. At present, it is considered not only the survival of extreme premature infants, but also the maintenance of their quality of life, since they are more likely to have sequelae at short, median and long time.

Keywords: Premature infant. Ethics. Extremely low birth weight infant. Child development.

1. Doutoranda alemos4@gmail.com 2. Doutoranda gisellemachado_mm@yahoo.com.br 3. Doutoranda aribeiro@hotlink.com.br 4. Doutoranda annachagas@hotmail.com 5. Doutoranda isis_suruagy@yahoo.com.br 6. Doutor josimario.bioetica@gmail.com Universidade Federal de Pernambuco, Recife, PE/Brasil. 
Os cuidados de saúde em situações de prematuridade extrema representam um dilema ético. A literatura corrente aborda estudos que discutem a decisão terapêutica tomada em situações de nascimento prematuro sob os pontos de vista cultural, religioso, financeiro, tecnológico e moral. Entre os profissionais de saúde (neonatologistas, obstetras, enfermeiros, fisioterapeutas, fonoaudiólogos etc.) envolvidos no cuidado direto com prematuros, definir o limite de idade gestacional, selecionado com base em evidências, para julgar quando o cuidado intensivo torna-se mandatório, opcional ou não traz garantia de sucesso, é tema polêmico e delicado.

Atualmente, considera-se que a decisão terapêutica entre utilizar cuidados de conforto e paliativos, dispensando cuidados intensivos, compete inteiramente aos pais esclarecidos e informados sobre as condições clínicas que envolvem a prematuridade extrema e as opções disponíveis de terapia ${ }^{1}$. Diversas publicações apresentam resultados claros e consistentes a respeito da decisão de parturientes e gestantes, a partir do processo de aconselhamento em situações envolvendo risco de morte dos bebês, e ao mesmo tempo compartilham recomendações específicas quanto a intervenção médica em casos de prematuridade extrema ${ }^{1-3}$.

Percebe-se que os índices de morbidade, aos poucos, têm substituído os de mortalidade em bebês prematuros nos diferentes limites de viabilidade ${ }^{4}$. Embora tenha sido observado significativo declínio da mortalidade dos nascidos vivos em extremos de prematuridade, a taxa de injúria neuroevolutiva permanece alta ${ }^{5-8}$. São esparsas as formas de avaliação da qualidade de serviços de cuidados neonatais relacionadas não só à saúde, mas à funcionalidade neuropsicomotora e à qualidade de vida dos pacientes ${ }^{9}$. Deve-se levar em consideração problemas relacionados a diferentes áreas de conhecimento, visando influenciar adequadamente as tomadas de decisão, transdisciplinar, em unidades de terapia intensiva neonatal ${ }^{10}$.

Dadas a variedade e a complexidade dos fatores envolvidos nas decisões acerca das intervenções terapêuticas em condições de prematuridade extrema, este estudo discute aspectos éticos dessa tomada de decisão considerando o limite de viabilidade, a prematuridade extrema e o desenvolvimento neuropsicomotor.

\section{Método}

O presente estudo é uma revisão crítica da literatura, e foram utilizadas as bases de dados PUBMED/Medline, Scielo e Lilacs. Foi feita busca pelos descritores "prematuro", "ética", "recém-nascido de extremo baixo peso", cadastrados nos Descritores em Ciências da Saúde (DeCS), e seus similares em inglês "premature infant", "ethics", "extremely low birth weight infant" (de acordo com o MESH); além dos unitermos "limite de viabilidade", "desenvolvimento neuropsicomotor" e "decisão terapêutica", considerando, ademais, seus respectivos cruzamentos.

Foram incluídos todos os estudos que abordaram o tema nos últimos dez anos, sem restrição de idioma. Foram excluídos trabalhos apresentados apenas em forma de resumo/abstract. Considerando que o assunto é vasto e polêmico, foram considerados os seguintes aspectos nesta revisão: limite de viabilidade, prematuridade extrema, desenvolvimento neuropsicomotor e considerações éticas sobre as decisões terapêuticas.

\section{Limite de viabilidade}

Existe variabilidade nos critérios de viabilidade de prematuros entre os países (desenvolvidos e em desenvolvimento) e depende também do tipo de centro de saúde que assiste a gestante e o recémnascido. Pode-se, entretanto, estabelecer o intervalo de possibilidade de viabilidade entre 22 e 26 semanas $^{11,12}$. Os dados disponíveis indicam que é extremamente improvável a sobrevivência de recémnascidos com menos de 23 semanas de gestação e peso menor que $500 \mathrm{~g}$ ao nascimento, com chance praticamente zero de sobrevivência ${ }^{13-15}$.

Estudo realizado por Doyle e colaboradores ${ }^{16}$ demonstrou que apenas $10 \%$ dos recém-nascidos de gestação de 22 semanas sobreviveram à internação em unidades de terapia intensiva, e nenhum permaneceu vivo por mais de seis meses. Resultados semelhantes foram encontrados por Markestad et al. ${ }^{17}$, para quem nenhum recém-nascido com idade gestacional inferior a 23 semanas sobreviveu. Os resultados desses estudos levantam a hipótese de que o não investimento terapêutico nesses recémnascidos seria a atitude eticamente correta. 
Para os bebês que nascem com mais de 23 semanas de gestação e peso maior que $500 \mathrm{~g}$, a sobrevivência e os resultados são incertos e difíceis de prever. Estas crianças estariam na chamada "zona cinzenta", e a tomada de decisão terapêutica deveria ser baseada na avaliação cuidadosa dos dados relativos ao pré-natal, sua idade gestacional, peso ao nascer e a condição clínica no momento do nascimento ${ }^{4}$. É preciso indicar, contudo, que também a delimitação da "zona cinzenta" difere entre os estudos, sendo considerado o período de nascimento entre 24 e 25 semanas ${ }^{18}$. No entanto, Parikh e colaboradores ${ }^{19}$ mostraram uma probabilidade geral de sobrevida, sem disfunção profunda, na faixa de $62 \%$ a $63 \%$ quando os recém-natos com 25 semanas de gestação são submetidos a cuidados intensivos. Por isso, em muitos centros, os cuidados intensivos têm sido mandatórios para crianças nascidas com 25 semanas de idade gestacional ${ }^{3}$. Contudo, em entrevista com médicos de países em desenvolvimento, foram considerados não-viáveis recém-nascidos com idade gestacional de até 25 semanas e peso ao nascimento de até $800 \mathrm{~g}^{12}$.

A maioria dos clínicos e investigadores concorda em aplicar o conceito da "zona cinzenta" como o mais coerente para definir os limites de viabilidade para grande parte da população de pacientes prematuros ${ }^{20}$. A classificação de pacientes pela "zona cinza" leva em consideração diversos fatores, como, por exemplo, a habilidade dos clínicos em classificar corretamente a idade gestacional da parturiente informada antes e imediatamente após o parto e a realização de consultas pré-natais regulares com o obstetra e a família.

O neonatologista deve participar do processo de tomada de decisão antes do parto e atender ao parto de todo neonato que se encontre próximo desse limite de viabilidade, já que abaixo do limite o recém-nascido é imaturo demais para ter chance razoável de sobrevivência e, em contrapartida, acima, é maior a oportunidade de sobrevivência sem disfunções severas. Além da idade gestacional, devem-se considerar outros fatores antes da tomada de decisão, por influenciarem positivamente o prognóstico do prematuro: maior peso para dada idade gestacional, gestação única, sexo feminino e exposição a corticoide antenatal ${ }^{18,21}$.

\section{Prematuridade extrema e desenvolvimento neuropsicomotor}

Apesar do progresso na qualidade dos cuidados perinatais, que representa diminuição na mortalidade, ainda existe elevado risco de injúria neurológica severa ${ }^{22}$. A possibilidade de sobrevivência sem disfunções e/ou deficiências significativas diminui com a idade gestacional, embora sejam heterogêneos os estudos sobre prevalência e desfecho das deficiências neurocognitivas e neuropsicomotoras associadas a prematuridade extrema ${ }^{11,18}$.

O prematuro deve ser avaliado de acordo com a classificação internacional de funcionalidade, que descreve aspectos das competências comportamentais, sócio-emocionais e adaptativas. As principais disfunções encontradas nos sobreviventes: atraso no desenvolvimento neuropsicomotor, decorrente de encefalopatia crônica não progressiva; cegueira; surdez; e alteração nas habilidades sociais e cognitivas ${ }^{23}$.

Experiências precoces desagradáveis podem modular a função endócrina e alterar o padrão de desenvolvimento dos circuitos neuronais, o que interfere nos sistemas sensitivo, motor e cognitivo. Há relatos na literatura de que recém-nascidos prematuros (RNPT) expostos ao ambiente estressante da unidade de terapia intensiva neonatal por tempo prolongado apresentam desenvolvimento cerebral e sensorial anormal, perda auditiva e problemas de linguagem ${ }^{24-27}$.

Sabe-se que prematuros extremos apresentam alterações em marcadores do desenvolvimento visual, mesmo sem lesão cerebral aparente em exames de imagem ${ }^{28}$. Eventos críticos e processos que ocorrem em fase importante de aquisições do sistema visual humano (da 20 à à 40 a semana de idade gestacional), como exposição a luminosidade excessiva ou oxigenoterapia, podem induzir a retinopatia da prematuridade, desde graus mais leves e tratáveis até graus mais severos, que culminam com cegueira. Além dessas sequelas, verificaramse também alterações do controle central do sistema visual em áreas como tálamo, córtex occipital, hipocampo, lobos parietal e frontal. Todos esses fatores predispõem a desenvolvimento visual inadequado, o que interfere na programação e no 
aprendizado de funções visuais, visuocognitivas e visuomotoras ${ }^{29-31}$.

Em longo prazo, são observadas diferenças nas disfunções em prematuros extremos de acordo com a faixa etária. As limitações funcionais mais comumente encontradas em idade pré-escolar envolvem atividades motoras, de autocuidado e de comunicação; em idade escolar, são identificados atrasos na educação em mais de $50 \%$ dos sobreviventes; na adolescência, estão presentes ainda limitações vocacionais e há relatos de distúrbios psiquiátricos ${ }^{32}$. Essas atividades necessitam de redes neurais de atenção bem estruturadas e funcionais, mas prematuros apresentam prejuízo no desenvolvimento precoce da atenção, que pode perdurar até a infância ${ }^{33}$, comprometendo etapas posteriores de aprendizado.

\section{Considerações éticas sobre as decisões tera- pêuticas}

Com o nascimento prematuro, a decisão de início e manutenção de cuidados intensivos ou paliativos é muito difícil e envolve uma série de aspectos éticos complexos. A instituição de cuidados avançados pode resultar na sobrevivência de neonatos severamente comprometidos do ponto de vista neuropsicomotor, cognitivo e afetivo; por outro lado, a não reanimação ou a não instituição de cuidados intensivos ao nascimento implica deixar o bebê morrer e pode suprimir a possibilidade de vida de um prematuro que se desenvolveria normalmente ${ }^{14}$. O dilema da equipe na tomada de decisão, sobre considerar prematuros viáveis ou não, reside no reconhecimento e na percepção de pessoalidade daquele recém-nascido, além de atribuições de valor à vida de acordo com fatores culturais e religiosos ${ }^{34}$.

O crescente avanço tecnológico na assistência à saúde e a necessidade de se buscar uma intervenção humanizada tornam imperativo refletir sobre as questões bioéticas no cotidiano das unidades neonatais. As novas concepções filosóficas e a falência do modelo biologista têm propiciado o repensar das práticas de cuidar, buscando ressaltar a visão humanista e existencial do cuidado ${ }^{35}$. Sublinhe-se nesse contexto que o objetivo da bioética é buscar beneficio e garantia da integridade do ser humano, tendo como fio condutor o princípio básico de proteção à dignidade humana ${ }^{36}$.

$\mathrm{Na}$ neonatologia, o princípio da autonomia é visto com ressalvas. Afinal, quem determina a escolha do que é o melhor ou mais apropriado ao recém-nascido hospitalizado: os profissionais ou seus pais? Considerando que a autonomia é o direito de a pessoa tomar suas próprias decisões e que os bebês não são capazes de expressar autonomia, os pais são legalmente autorizados a dar consentimento para que seja feito um tratamento ${ }^{13}$. Nesse sentido, defendem-se discussões e diálogos contínuos entre a equipe de saúde e os representantes legais para que sejam tomadas decisões acerca dos procedimentos a serem empregados no tratamento do neonato ${ }^{36}$.

Manter artificialmente as funções vitais sem expectativas razoáveis de recuperação pode prolongar o sofrimento do paciente e de seus familiares, o que chega a atentar contra a própria dignidade do enfermo. Isso nem sempre representa o equilíbrio favorável entre riscos e benefícios e implica em qualidade de vida muito baixa, que pode culminar ainda com a exclusão de pacientes mais necessitados e viáveis ${ }^{37}$, devido à carência de recursos para atender a todos os prematuros. O que se tem discutido mais recentemente é a terapêutica voltada a cuidados paliativos em prematuros abaixo da zona cinzenta, que inclui alívio de dor e sofrimento para o recém-nascido e, em relação aos familiares, apoio psicológico e orientação em relação a próximas gestações, em particular nos casos de malformações congênitas ${ }^{38}$.

Os profissionais de saúde devem considerar ainda toda a rede de apoio social e suporte às necessidades do prematuro extremo que sobreviveu, pois, para melhorar o prognóstico do desfecho funcional daqueles com disfunções leves a moderadas, é preciso otimizar a participação da comunidade e o suporte à família ${ }^{32}$, dado que programas de intervenção precoce parecem ser positivos a curto e médio prazos ${ }^{39}$. A equipe pode também buscar se certificar da facilidade de acesso desses pacientes a centros especializados, com programas de acompanhamento após a alta hospitalar implementados por equipe interdisciplinar, voltados a atender aqueles com deficiências maiores.

\section{Considerações finais}

Inovações no suporte avançado de vida, maior especialização dos profissionais de saúde, frequência e adequação dos exames pré-natais, progresso no diagnóstico e intervenção precoce de acometimentos perinatais corrigíveis são processos que permitem a sobrevivência de recém-nascidos com idade gestacional e peso ao nascer extremamente baixos, alargando os limites de viabilidade. 
Entretanto, diante dessa possibilidade, tem-se visto que grande número de prematuros extremos está exibindo problemas neurocomportamentais, como diminuição do alcance cognitivo, transtorno de atenção e hiperatividade na infância além de transtornos psiquiátricos na adolescência, mesmo na ausência de encefalopatia não progressiva, implicando graus variados de limitação neurocognitiva, físicofuncional e dependência. Isso suscita a questão sobre a importância da valorizar não só a sobrevivência desses prematuros, mas também a manutenção de sua qualidade de vida, vez que eles são mais predispostos a sequelas em curto, médio e longo prazos. Ainda há dificuldade em definir o nível limítrofe de prematuridade que guia a tomada de decisão em relação à terapêutica que deve ser adotada (paliativa ou intensiva), principalmente quando se consideram os recursos disponíveis na unidade que receberá o prematuro extremo. É importante manter o envolvimento da família no processo decisório, a fim de que não seja extinta a possibilidade de vida plena para um prematuro possivelmente viável.

\section{Referências}

1. Kaempf JW, Tomlinson MW, Campbell B, Ferguson L, Stewart VT. Counseling pregnant women who may deliver extremely premature infants: medical care guidelines, family choices, and neonatal outcomes. Pediatrics. 2009 jun;123(6):1.509-15.

2. Committee on Fetus and Newborn. Noninitiation or withdrawal of intensive care for high-risk newborns. Pediatrics. 2007 fev;119(2):401-3.

3. Pignotti MS, Donzelli G. Perinatal care at the threshold of viability: an international comparison of practical guidelines for the treatment of extremely preterm births. Pediatrics. 2008 jan;121(1):e193-8.

4. Seri I, Evans J. Limits of viability: definition of the gray zone. J Perinatol. 2008 maio;28 (Suppl 1):S4-8.

5. Marlow N, Wolke D, Bracewell MA, Samara M, EPICure Study Group. Neurologic and developmental disability at six years of age after extremely preterm birth. N Engl J Med. 2005 jan 6;352(1):9-19.

6. Vincer MJ, Allen AC, Joseph KS, Stinson DA, Scott H, Wood E. Increasing prevalence of cerebral palsy among very premature infants: a population-based study. Pediatrics. 2006 dez;118(6):e1.621-6.

7. Wilson-Costello D, Friedman H, Minich N, Siner B, Taylor G, Schluchter M et al. Improved neurodevelopmental outcomes for extremely low birth weight infants in 2000-2002. Pediatrics. 2007 jan;119(1):37-45.

8. Poon WB, Ho SKY, Yeo CL. Short- and long-term outcomes at 2, 5, and 8 years old for neonates at borderline viability: an 11-year experience. Ann Acad Med Singapore. 2013 jan;42(1):7-17.

9. Hack M. Consideration of the use of health status, functional outcome, and quality-of-life to monitor neonatal intensive care practice. Pediatrics. 1999 jan;103(1 Suppl E):319-28.

10. Simard M, Gagné AM, Lambert RD, Tremblay Y. A transdisciplinary approach to the decisionmaking process in extreme prematurity. BMC Res Notes. 2014 jul 14;7:450-8.

11. Lorenz JM. The outcome of extreme prematurity. Semin Perinatol. 2001 out;25(5):348-59.

12. Charafeddine L, Ammous F, Kayle M, Arawi T. Survival at the threshold of viability: a nationwide survey of the opinions and attitudes of physicians in a developing country. Paediatr Perinat Epidemiol. 2014 maio;28(3):227-34.

13. Chervenak FA, McCullough LB, Levene MI. An ethically justified, clinically comprehensive approach to peri-viability: gynecological, obstetric, perinatal and neonatal dimensions. J Obstet Gynaecol. 2007 jan;27(1):3-7.

14. Hack M, Fanaroff AA. Outcomes of children of extremely low birth weight premature infants in the 1990s. Early Hum Dev. 1999;53:195-218.

15. García-Muñoz FR, García-Alix AP, García Hernández JA, Figueras JA. Morbidity and mortality in newborns at the limit of viability in Spain: a population-based study. An Pediatr (Barc). 2014 jun;80(6):348-56.

16. Doyle LW, the Victorian Infant Collaborative Study Group. Neonatal intensive care at borderline viability: is it worth it? Early Hum Dev. 2004 nov;80(2):103-13.

17. Markestad T, Kaaresen PI, Rønnestad A, Reigstad H, Lossius K, Medbø Set al. Early death, morbidity, and need of treatment among extremely premature infants. Pediatrics. 2005 maio;115(5):1.28998.

18. Moriette G, Rameix S, Azria E, Fournié A, Andrini P, Caeymaex L. et al. Very premature births: Dilemmas and management. Part 1. Outcome of infants born before 28 weeks of postmenstrual age, and definition of a gray zone. Arch Pediatr. 2010 maio;17(5):518-26.

19. Parikh DO, Arnold C, Langer J, Tyson JE. Evidence-based treatment decisions for extremely preterm newborns. Pediatrics. $2010 \mathrm{abr} ; 125(4): 813-6$.

20. Singh J, Fanaroff J, Andrews B, Caldarelli L, Lagatta J, Plesha-Troyke S et al. Resuscitation in the "gray zone" of viability: determining physician preferences and predicting infant outcomes. Pediatrics. 2007 set;120(3):519-26.

21. Rocha G, Guimarães H. On the limit of viability extremely low gestational age at birth. Acta Med Port. 2011 dez;24(Suppl 2):181-8. 
22. Shah PS, Sankaran K, Aziz K, Allen AC, Seshia M, Ohlsson A et al. Outcomes of preterm infants $<29$ weeks gestation over 10-year period in Canada: a cause for concern? J Perinatol. 2012 fev;32(2):132-8.

23. Msall ME, Park JJ. The spectrum of behavioral outcomes after extreme prematurity: regulatory, attention, social, and adaptive dimensions. Semin Perinatol. 2008 fev;32(1):42-50.

24. Bremmer $P$, Byers JF, Kiehl E. Noise and the premature infant: physiological effects and practice implications. J Obstet Gynecol Neonatal Nurs. 2003 jul-ago;32(4):447-54.

25. Brown G. NICU noise and the preterm infant. Neonatal Netw. 2009;28(3):165-73.

26. Perlman JM. Neurobehavioral deficits in premature graduates of intensive care: potential medical and neonatal environmental risk factors. Pediatrics. 2001 dez;108(6):1.339-48.

27. Williams AL, Sanderson M, Lai D, Selwyn BJ, Lasky RE. Intensive care noise and mean arterial blood pressure in extremely low-birth-weight neonates. Am J Perinatol. 2009 maio;26(5):323-9.

28. Atkinson J, Braddick O. Visual and visuocognitive development in children born very prematurely. Prog Brain Res. 2007;164:123-49.

29. Braddick O, Atkinson J, Wattam-Bell J. VERP and brain imaging for identifying levels of visual dorsal and ventral stream function in typical and preterm infants. Prog Brain Res. 2011;189:95111.

30. Graven SN. Early visual development: implications for the neonatal intensive care unit and care. Clin Perinatol. 2011 dez;38(4):671-83.

31. Ramenghi LA, Ricci D, Mercuri E, Groppo M, De Carli A, Ometto A et al. Visual performance and brain structures in the developing brain of pre-term infants. Early Hum Dev. 2010 jul;86 (Suppl 1):73-5.

32. Msall ME, Tremont MR. Measuring functional outcomes after prematurity: developmental impact of very low birth weight and extremely low birth weight status on childhood disability. Ment Retard Dev Disabil Res Rev. 2002;8(4):258-72.

33. Van der Weijer-Bergsma E, Wijnroks L, Jongmans MJ. Attention development in infants and preschool children born preterm: a review. Infant Behav Dev. 2008 set;31(3):333-51.

34. Christoffersen-Deb A. Viability: a cultural calculus of personhood the beginnings of life. Med Anthropol Q. 2012 dez;26(4):575-94.

35. Kletemberg DF, Mantovani MF, Lacerda MR. Entre a teoria e as práticas do cuidar: que caminho trilhar? Cogitare Enferm. 2004;9(1):94-9.

36. Gaiva MAM. O cuidar em unidades de cuidados intensivos neonatais: em busca de um cuidado ético e humanizado. Cogitare Enferm. 2006;11(1):61-6.

37. Ribeiro CDM, Rego S. Bioética clínica: contribuições para a tomada de decisões em unidades de terapia intensiva neonatais. Ciênc saúde coletiva. 2008 dez;13 (Suppl 2):2.239-46.

38. Schramm FR. Cuidados paliativos: aspectos filosóficos. Rev Bras Cancerol. 1999;45(4):57-63.

39. Spittle A, Orton J, Anderson P, Boyd R, Doyle LW. Early developmental intervention programs post-hospital discharge to prevent motor and cognitive impairments in preterm infants. Cochrane Database of Systematic Reviews. 2012 dez;12:1-103.

\section{Participação dos autores}

Andrezza de Lemos Bezerra, Giselle Machado Magalhães Moreno, Adriana Carla Costa Ribeiro Clementino, Anna Paula Chaves Chagas e Isis Suruagy Moura participaram da pesquisa e da elaboração do texto. Josimário Silva orientou e revisou o trabalho.

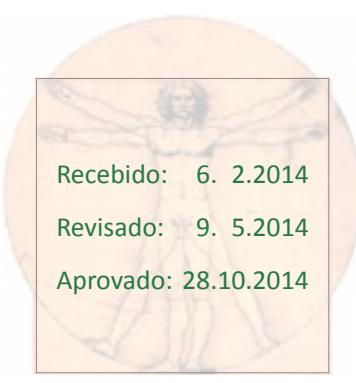

